\title{
Acquiring experience in pathology predominantly from what you see, not from what you read: the HIPON e-learning platform
}

This article was published in the following Dove Press journal:

Advances in Medical Education and Practice

8 June 2015

Number of times this article has been viewed

\section{Olga Riccioni $i^{1,2}$ \\ Charalambos Vrasidas ${ }^{3}$ \\ Luka Brcic ${ }^{4}$ \\ Goce Armenski ${ }^{5}$ \\ Sven Seiwerth ${ }^{4}$ \\ Annemieke Smeets ${ }^{6}$ \\ J Han JM van Krieken ${ }^{6}$ \\ Andreas C Lazaris'}

'Ist Department of Pathology, School of Medicine, The National and Kapodistrian University of Athens, Athens, Greece;

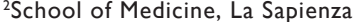

University of Rome, Rome, Italy; ${ }^{3}$ Centre

for the Advancement of Research and

Development in Educational Technology

LTD, Nicosia, Cyprus; ${ }^{4}$ Institute of

Pathology, School of Medicine, University

of Zagreb, Zagreb, Croatia; ${ }^{5}$ Faculty

of Computer Science and Engineering,

Saints Cyril and Methodius University,

Skopje, FYROM/Republic of Macedonia;

${ }^{6}$ Department of Pathology, Radboud

University Nijmegen Medical Center,

Nijmegen, the Netherlands

\section{Video abstract}

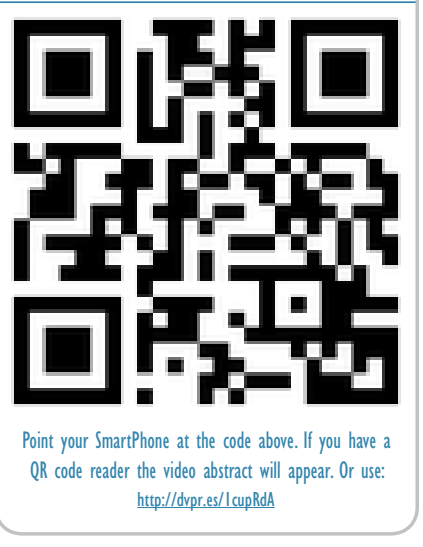

Correspondence: Olga Riccioni

School of Medicine, La Sapienza University of Rome, Piazzale Aldo Moro 5, 00185 , Rome, Italy

Tel +393319343242

Email olga.riccioni@outlook.com
Abstract: It is indisputable that nowadays one of the hardest and most important tasks in medicine and especially in medical education, is the conversion of the extensive amount of available data, into medical experience, after a proper analysis. A project under the title "ICT (Information and Communication Technology) eModules on HistoPathology: a useful online tool for students, researchers and professionals - HIPON", co-financed by the Lifelong Learning Program of the Education, Audiovisual and Culture Executive Agency (EACEA), The Commission of the European Union, has been launched at the beginning of 2013. HIPON's purpose is not to provide just another pathology website atlas, but to convey professional experience and thinking in pathology. HIPON has resulted in a well-structured and user-friendly, open resource, multi-language, e-learning platform which, taking advantage of modern image technology, offers medical students, researchers, and professionals a valuable teaching instrument so that they can acquire professional experience in pathology. The mid-term report of HIPON has been favorably evaluated by the EACEA experts who appreciated the potential of our teaching tool in providing the opportunity and the means to acquire medical experience. Through the use of virtual slides, educative videos and microscopic, high resolution, marked images accompanied by relevant questions and answers, HIPON project aims to make end-users able to think as experienced pathologists and become highly efficient in correlating pathologic data with other clinical-laboratory information.

Keywords: international medical education, website, educational technology, case study

\section{Introduction}

Nowadays, pathology represents a major diagnostic field in medicine. Through the analysis of tissue samples, ie, biopsies and surgical resections, it allows medical doctors to exclude or confirm a suspected clinical diagnosis, such as cancer, and even to identify the presence of unsuspected concurrent diseases. The pathological examination of specimens under the microscope may be supported by further, tissue-based laboratory tests such as those making use of molecular biology techniques. A high level of competence in recognizing patterns of injury when tissue specimens are approached, and in correlating the essential pathological data with other clinical-laboratory information is of vital importance to ensure that the correct diagnosis is made.

The role that pathologists have in patient care is indeed crucial, since they are responsible for documenting fully the diagnostic evidence tissue samples can provide. For example, the pathologist's interpretation of a tumor specimen is critical to establish the diagnosis of a benign or a malignant tumor, to distinguish between distinct histogenetic types of neoplasia, as well as to estimate the grade and the stage of the malignant neoplastic disease. All information provided by pathologists determines patients' prognosis and efficient treatment selection. 
It may take over 14 years to become a fully qualified pathologist. Being part of fundamental medical knowledge, pathology is currently taught, mainly on a theoretical basis, from the undergraduate level of medical studies. Attending lectures and taking advantage of excellent textbooks and atlases, students are supposed to learn how to recognize state of disease and describe main patterns of tissue injury. In their professional life, pathologists and researchers are requested to evaluate microscopic diagnostic features in patients' tissue sections so that a definite diagnosis is set.

In the immense field of modern pathology, an extensive amount of data is available; so, as many practical skills as possible need to be developed by medical professionals. After discussing and implementing teaching strategies in pathology and evaluating students' learning, teachers are developing new-style pathology courses. ${ }^{1}$

In recent years, e-learning tools have been developed to supplement traditional text-based materials ${ }^{2}$ and are being progressively included into medical education, ${ }^{3}$ due to their teaching potential; the relevant educational methods, in which students are requested to be active searchers of knowledge instead of passive recipients, ${ }^{4}$ have been shown to be associated with increased learning outcomes, with regard to various areas of health and medical education.,

It is indisputable that simulation is a promising pedagogical tool in medicine. ${ }^{7}$ A new teaching approach based on online case studies and interactive discussions, has already been considered successful in teaching pathophysiology. ${ }^{8}$ The motivation to learn is greatly improved by the study of cases; ${ }^{9}$ the latter makes pathology easier to understand and, furthermore, in this way, students can relate knowledge to a real world context and their future profession. ${ }^{10}$

The main characteristics of the modern pathology module consist of digitalized pathology images combined with web-based delivery of compact and guided learning courses. If we consider that changes are occurring from traditional microscopy towards examination of scanned slides, we understand the importance of a teaching approach based on new technological devices. ${ }^{11}$

\section{The HIPON project}

Based on the above, an educational project on histopathology entitled "ICT eModules on HistoPathology: a useful online tool for students, researchers and professionals - HIPON", co-financed as a key activity 3/ICT project of the Lifelong Learning Program of the Education, Audiovisual and Culture Executive Agency (EACEA), The Commission of the European
Union (reference number: 531203-LLP-1-2012-1-GR-KA3KA3MP) has been launched at the beginning of 2013.

HIPON integrates the new virtual microscope/e-learning environment in multiple educational units/chapters of pathology, specifically tailored to the needs of students (undergraduate, graduate, and postgraduate students), researchers, and professionals.

The project has hopefully resulted in a well-structured and user-friendly, open resource, multi-language e-learning platform with brief texts and extended microscope, high quality image collections, supported by educational videos and relevant virtual slides. High pedagogical experience is included in the courses so as to convey a way of thinking and experience, instead of mere information.

\section{HIPON purpose}

HIPON's purpose is not to provide just another pathology website atlas, among the excellent existing ones, but to pass on professional experience and thinking in pathology. Medical students are required to learn and retain vast amounts of knowledge on the path to becoming physicians; ${ }^{2}$ nowadays one of the hardest and most important tasks in medical education is the conversion of the extensive amount of available data into medical experience.

Building and putting together knowledge, skills, and attitudes is one of the project's biggest aims. Authentic learning is improved by the use of case studies and real world problem solving. Learning authentically implies that users are confronted with simulations of their present or future professional practice and are therefore stimulated to develop proper competencies. ${ }^{12}$

Aristotle once said, "For the things we have to learn before we can do them, we learn by doing them". Based on preliminary testing results, HIPON focusing on experiential learning appears to contribute to medical education.

\section{HIPON evaluation}

The mid-term report of our project has recently been evaluated by the EACEA experts who appreciated the potential of our teaching tool in providing the opportunity and the means to acquire medical experience.

Discussing the value of e-learning, a major issue is the potential difference in material quality, communication skills, and digital setup. ${ }^{13}$ According to EACEA quality assurance, HIPON's evaluation has been considered as most satisfactory; the mid-term report acknowledges significant and valuable project results. 
In detail, several criteria, such as objectives, results and products and coherence between work plan and activities carried out during the life of the project, have been considered. With regard to the former, the EACEA evaluators have asserted that:

The HIPON initiative remains highly relevant to the program in its very good address to supporting students and developing professionals in histo- and cyto-pathology. The project activities are completely in accordance with its aims. The HIPON architecture is highly appropriate to the project objectives, and can be seen to explicitly respond to the features evident in the needs analysis. Similarly, the initial content responds to user needs.

Going on, for each of the evaluated criteria, comments have been made and a score was assessed; the global HIPON score hovered around the level of $90 \%$. In conclusion, an overall evaluation was provided reporting "no significant weakness" and outlining HIPON "strong points such as the realistic approach to user engagement, the good potential for uptake by a significant user community, and the project directly addressing a health education area of high public interest with a practical and achievable solution".

HIPON "end-product" is currently being tested by groups of students and professionals in all partners' countries and learners' perspectives are being assessed using both quantitative and qualitative measures. On the completion of the initial phase of the 10 -month HIPON testing period in the six involved countries, test users' evaluations appear to be in line with the above considerations.

\section{HIPON structure}

In HIPON module A, basic aspects of pathology are presented through representative virtual slides with educational annotations and assignments, often supplemented by still image files with markings, the aim of which is to stress the most important diagnostic morphological findings. After this first module dealing with general pathology aspects such as inflammation and neoplasia, a second one, on systemic pathology, reflects the participating pathologists' professional experience on the various organs - systems of the human body in its units/chapters.

\section{Overview sections}

Every Systemic Pathology chapter begins with an overview where the objectives of the chapter are defined and some basic guidelines are provided on how the relevant tissue samples should be examined under the microscope; the basic normal tissue architecture is highlighted and basic morphologic patterns of injury are analyzed in appropriate brief texts accompanying multiple, high resolution, enlargeable images (Figure 1). The text and the extended microscope image collections are supported by educational videos and relevant virtual slides (Figure 2) that help users consolidate the acquired knowledge. Glossary terms, their definitions, relevant resources, and web links are provided.

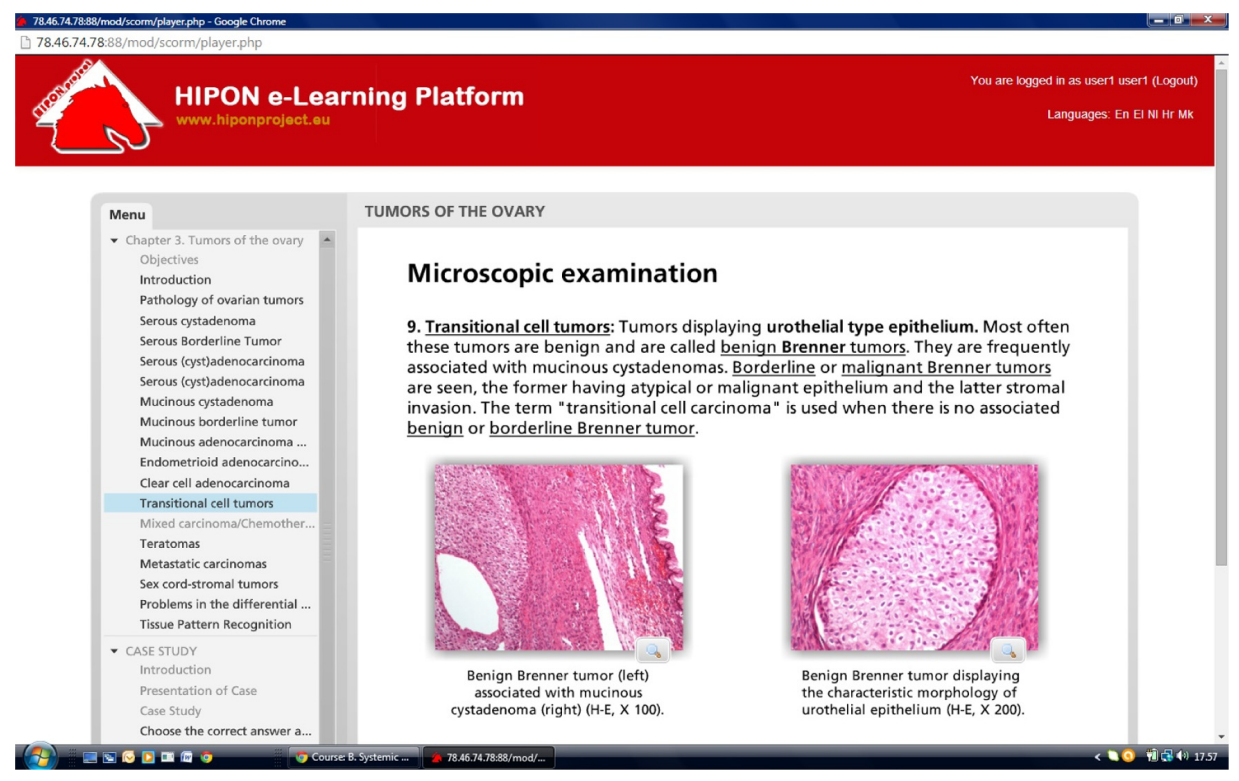

Figure I An example of a HIPON chapter overview.

Note: HIPON - ICT eModules on HistoPathology: a useful online tool for students, researchers and professionals. 


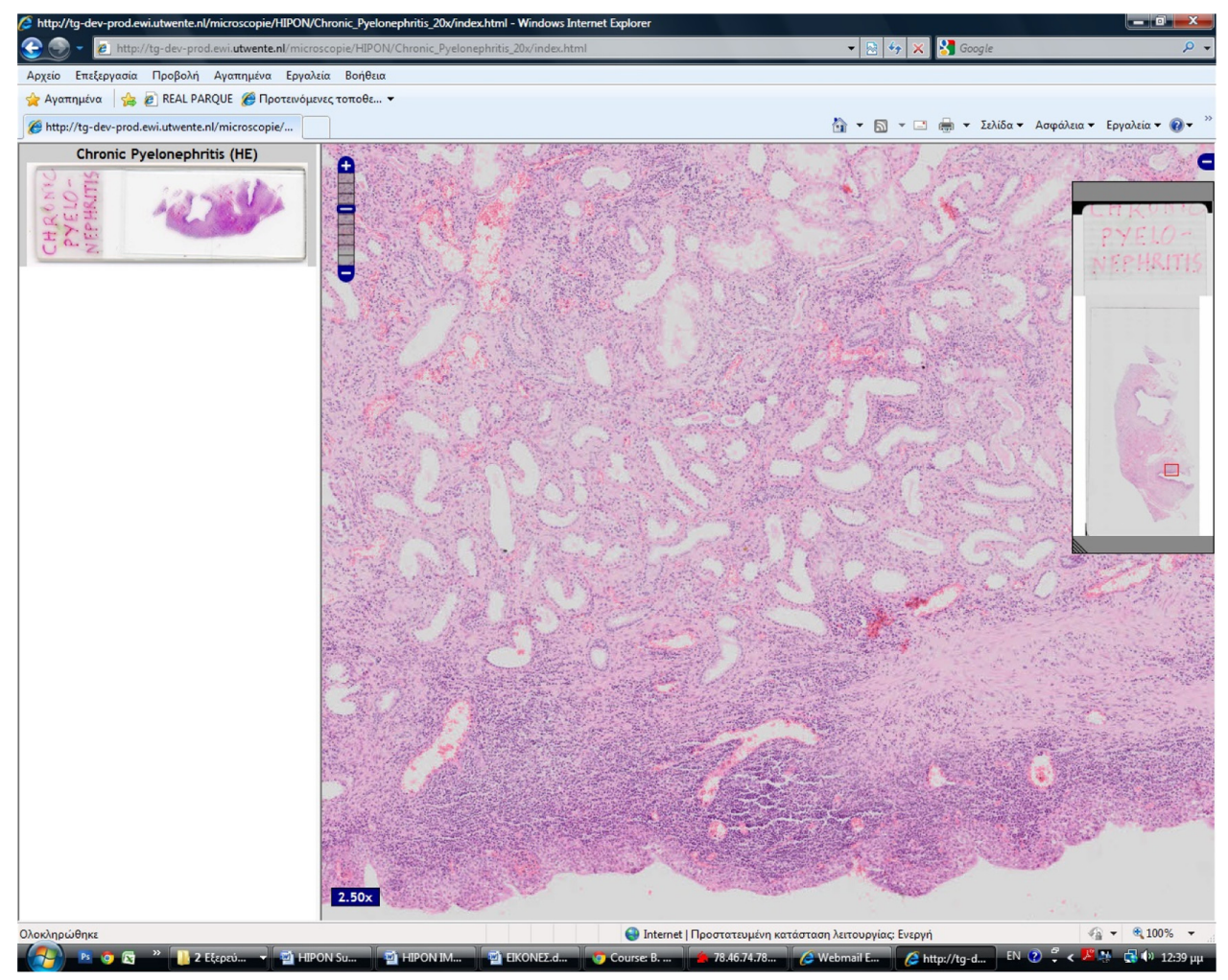

Figure 2 HIPON virtual slide of chronic pyelonephritis.

Note: HIPON - ICT eModules on HistoPathology: a useful online tool for students, researchers and professionals.

\section{Case studies}

The case study files that follow in each chapter, the most important and innovative section of the project, present the correct diagnostic procedure ie, the same process a pathologist follows when a biopsy is examined in every day practice, step by step. The aim is to help users familiarize themselves with the process of using all information received from the overview section and image web links, in order to approach a tissue specimen derived from a specific patient responsibly, and reach the correct pathologic diagnosis

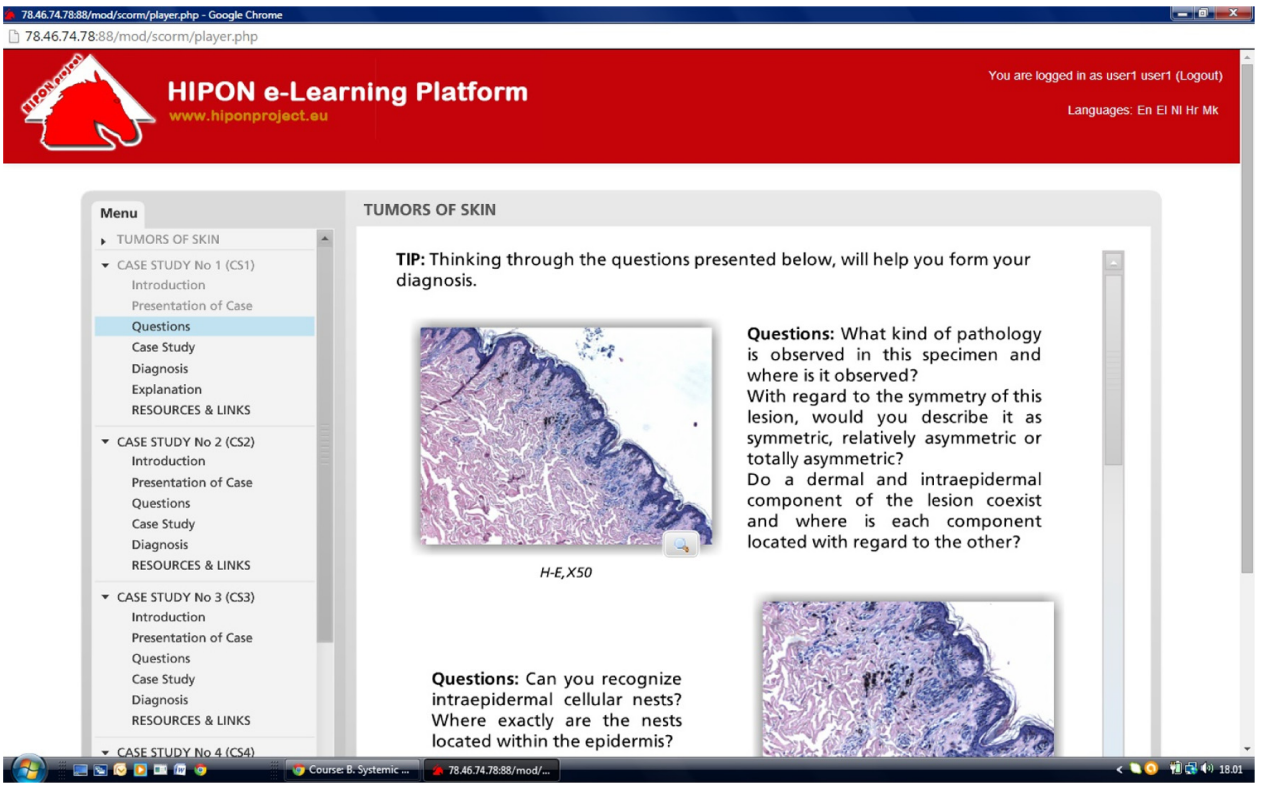

Figure 3 Presentation of a dysplastic nevus case in HIPON Skin neoplasms chapter.

Note: HIPON - ICT eModules on HistoPathology: a useful online tool for students, researchers and professionals. 


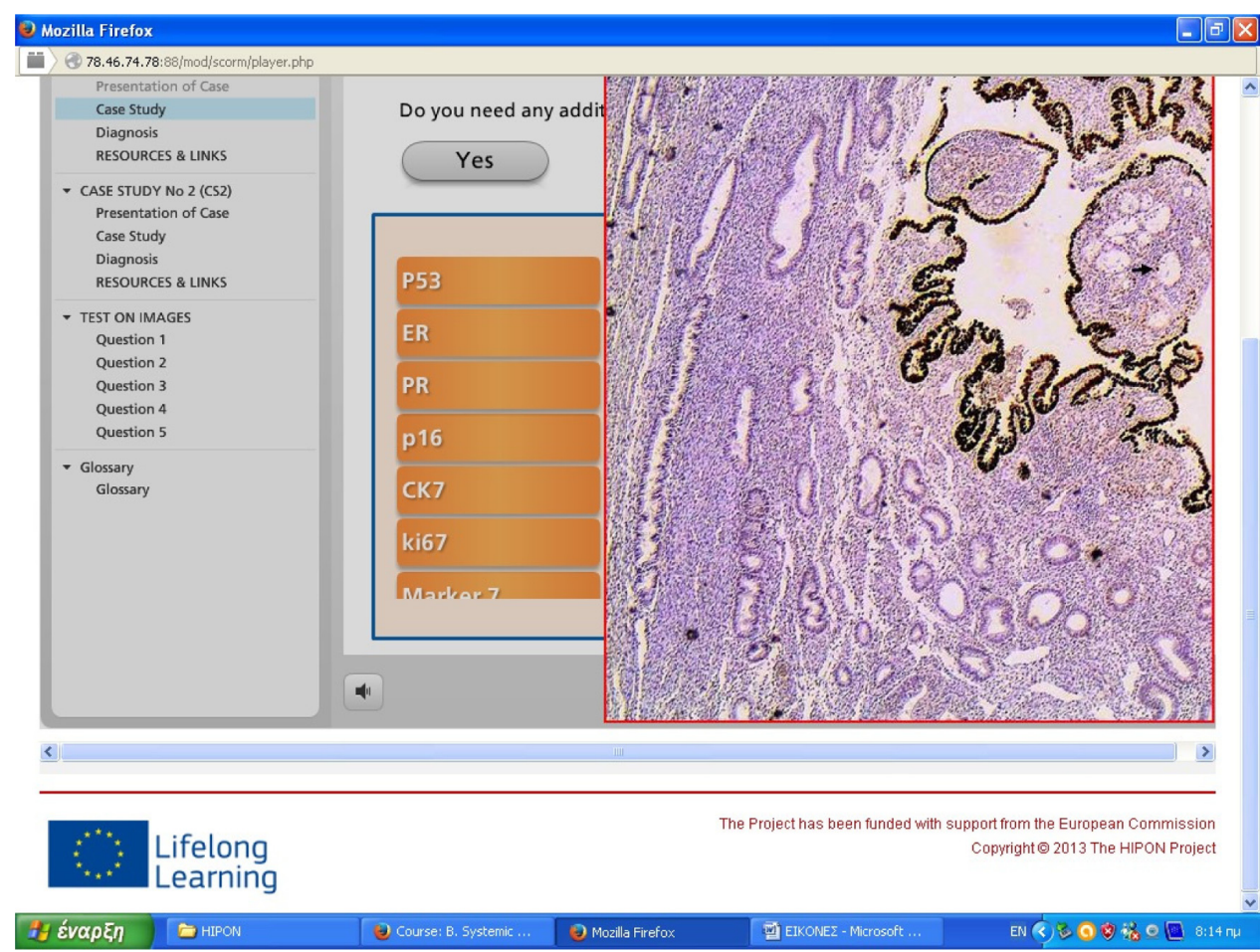

Figure 4 Option of immunohistochemical stain, helpful for defining the correct diagnosis, from a case presentation of an endometrial tumor in HIPON Uterus chapter. Notes: HIPON - ICT eModules on HistoPathology: a useful online tool for students, researchers and professionals. Copyright @ 2013 The HIPON Project. Reproduced with permission.

in the end. This approach is based on the methodology of an experienced pathologist and makes use of the practical advice he can offer on how to reach the correct diagnosis ie, primarily take into account the basic pattern of the lesion (and do not pay primary attention to confusing details), then look for any specific diagnostic features and evaluate their significance, correlating them with underlying mechanisms of disease.

As an example of the application of our educational concept, we can mention a case study of a skin neoplasm in the relevant HIPON Systemic Pathology chapter. At the beginning, a real clinical case is presented; images from the

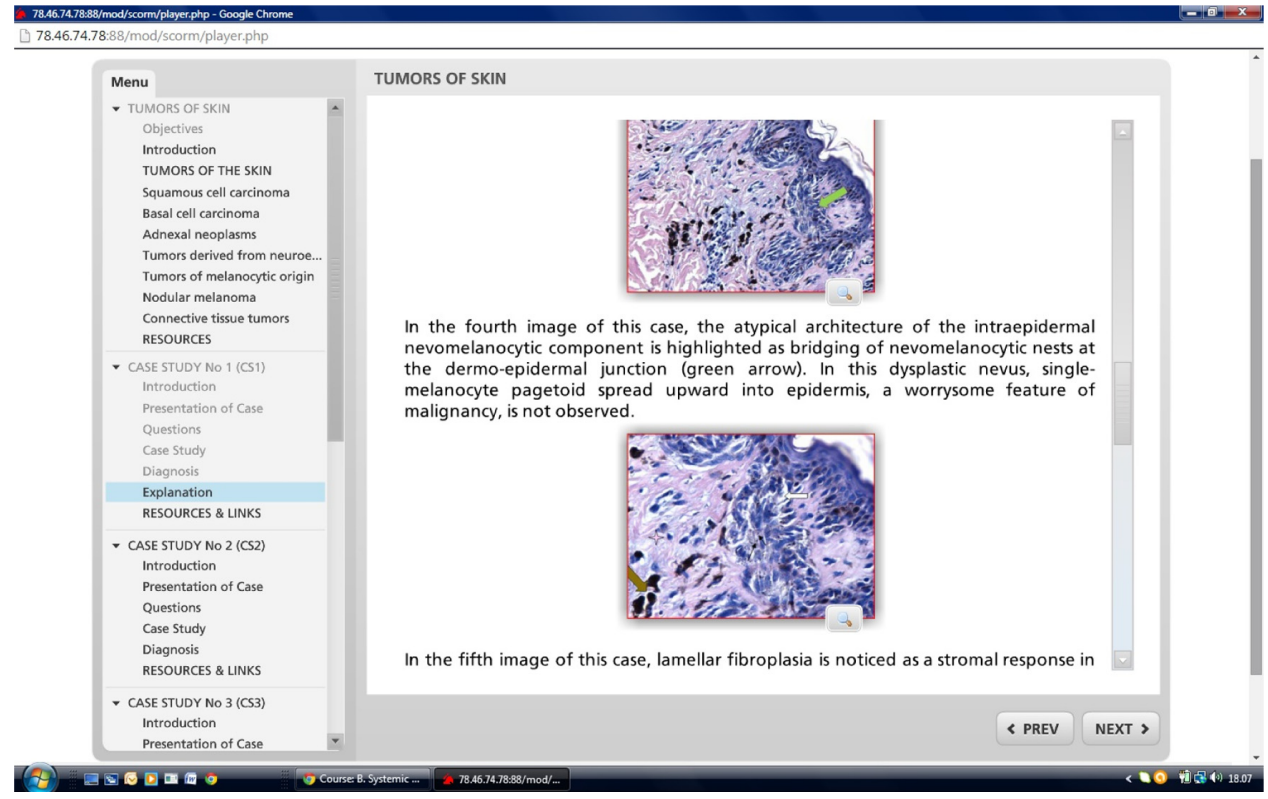

Figure 5 Image-by-image explanation of the dysplastic nevus diagnosis in the relevant HIPON chapter.

Note: HIPON - ICT eModules on HistoPathology: a useful online tool for students, researchers and professionals. 


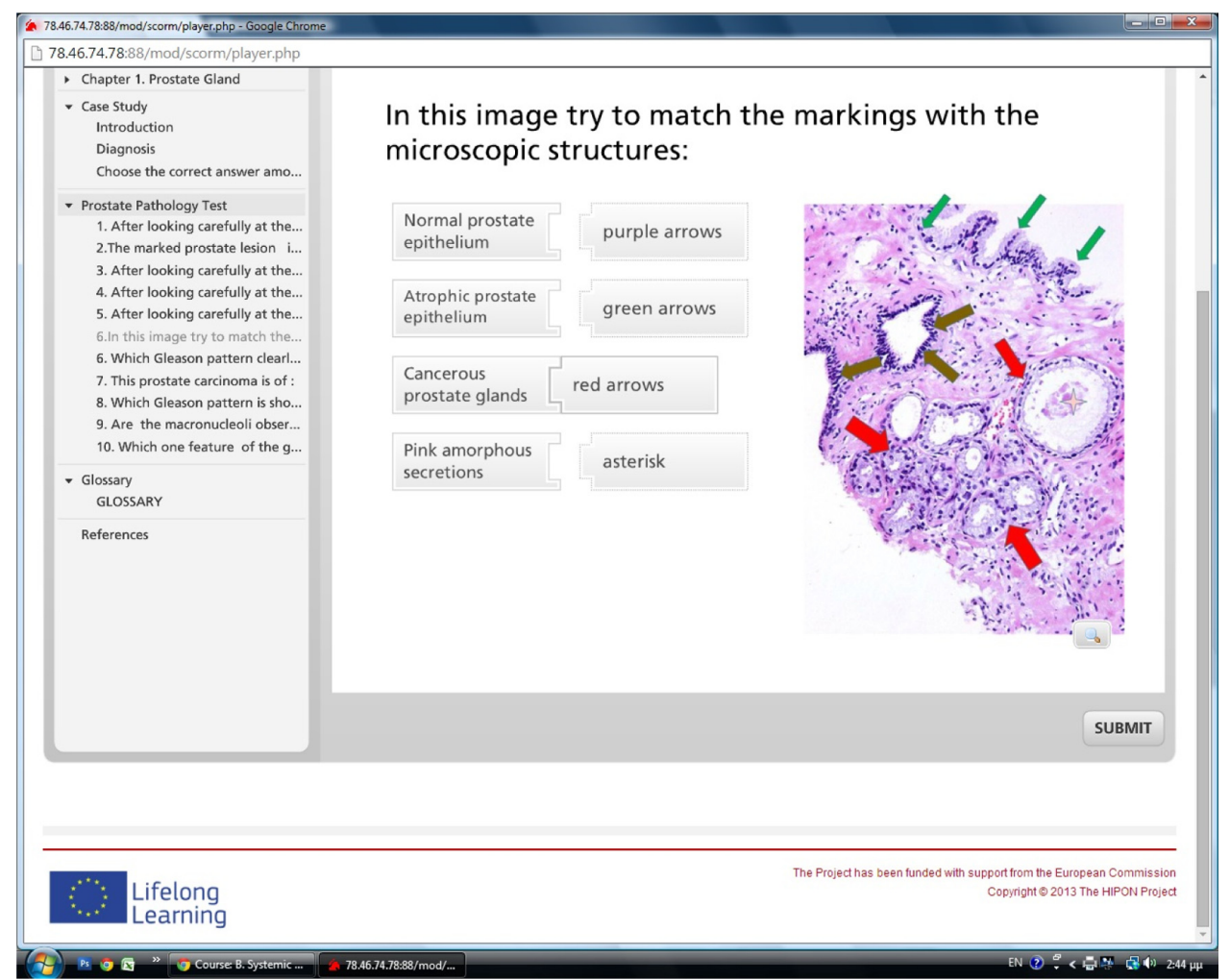

Figure 6 An example of an image-based question on prostate gland pathology in the relevant HIPON chapter.

Notes: HIPON - ICT eModules on HistoPathology: a useful online tool for students, researchers and professionals. Copyright @ 2013 The HIPON Project. Reproduced with permission.

real respective biopsy are provided, accompanied by questions which the user should try to answer (Figure 3), or, in more difficult case studies, accompanied by brief legends and comments. Their aim is to help users identify the most important diagnostic morphological findings (ie, as far as the case of dysplastic nevus is concerned, architectural distortion of the junctional component, nuclear pleomorphism, maturation of the dermal component, mitotic activity assessment) which will help them form their diagnosis. As in every day practice, if considered useful, the users can optionally ask for additional information (ie, immunohistochemistry [Figure 4], histochemistry, further laboratory data, more detailed patient history, and imaging) in order for the correct diagnosis to be achieved; the latter data must frequently be chosen among a number of alternatives proposed to the users, some of which are of no diagnostic value and should thus be disregarded. A detailed explanation of the diagnosis comes next; the means by which all previously provided data lead to the final diagnosis, is clarified. The images, already shown at the beginning of case study presentation, are displayed again; this time the answers to the respective questions are provided and their connection to the diagnosis is highlighted (Figure 5). The diagnostic significance of any additional information required is explained and some basic guidelines on differential diagnosis are provided. Relevant resources, web links and, furthermore, some practical tips on the correct diagnostic procedure are provided in order to consolidate the acquired knowledge.

\section{Image-based tests}

The degree of consolidation of the users' diagnostic experience can be estimated by an image-based test, present at the section that closes every chapter. We can mention as an example, the image-based test of the Prostate gland chapter. In this test, the users have to identify specific histological features on their own by answering single-answer or multiplechoice, image-based questions as well as by matching the microscopic features shown in images with the relevant morphological terms (Figure 6). Then, a review of the test is provided and, in this chapter, it is complemented by an original PowerPoint link in which the answers to the test questions are thoroughly explained.

\section{Conclusion}

By doing all this, HIPON aims to make end-users able to think as experienced pathologists and become highly competent in approaching tissue specimens, recognizing patterns of injury and correlating them with both the 
underlying mechanisms of disease and the clinical data. HIPON thus claims to be in harmony with all four aims set by the "European Union strategic framework for education and training" ie, making lifelong learning and mobility a reality, improving the quality and efficiency of education and training, promoting equity and social cohesion and enhancing creativity and innovation, at all levels of education and training.

\section{Acknowledgment}

All authors express their gratitude to the staff of AVMap Group of Companies for their administrative support.

\section{Disclosure}

The authors report no conflicts of interest in this work.

\section{References}

1. Marshall R, Cartwright N, Mattick K. Teaching and learning Pathology: a critical review of the English literature. Med Educ. 2004;38(3): 302-313.

2. Yang A, Goel H, Bryan M, et al. The Picmonic $\left({ }^{\circledR}\right)$ Learning System: enhancing memory retention of medical sciences, using an audiovisual mnemonic Web-based learning platform. Adv Med Educ Pract. 2014;5: $125-332$.
3. Kim S, Song SM, Yoon YI. Smart learning services based on smart cloud computing. Sensors (Basel). 2011;11(8):7835-7850.

4. Alur P, Fatima K, Joseph R. Medical teaching websites: do they reflect the learning paradigm? Med Teach. 2002;24(4):422-424.

5. Lakshmanan A, Leeman KT, Brodsky D, Parad R. Evaluation of a web-based portal to improve resident education by neonatology fellows. Med Educ Online. 2014;19:24403.

6. Cook D, Levinson AJ, Garside S, Dupras DM, Erwin PJ, Montori VM. Instructional design variations in internet-based learning for health professions education: a systemic review and meta-analysis. Acad Med. 2010;85(5):909-922.

7. Carron PN, Trueb L, Yersin B. High-fidelity simulation in the nonmedical domain: practices and potential transferable competencies for the medical field. Adv Med Educ Pract. 2011;2:149-155.

8. Van Dijiken PC, Thevoz S, Jucker-Kupper P, Feihl F, Bonvin R, Waeber B. Evaluation of a case-based interactive approach to teaching pathophysiology. Med Teach. 2008;30(5):e131-e136.

9. Dacre JE, Fox RA. How should we be teaching our undergraduates? Ann Rheum Dis. 2000;59(9):662-627.

10. Weurlander M, Masiello I, Soderberg M, Wernerson A. Meaningful learning: students' perceptions of a new form of case seminar in pathology. Med Teach. 2009;31(6):248-253.

11. Hamilton PW, Wang Y, McCullough SJ. Virtual microscopy and digital pathology in training and education. APMIS. 2012;120(4):305-315.

12. Herrington J, Kervin L. Authentic learning supported by technology: 10 suggestions and cases of integration in classrooms. EMI Educ Media Int. 2007;44(3):219-236.

13. Worm BS, Jensen K. Does peer learning or higher levels of e-learning improve learning abilities? A randomized controlled trial. Med Educ Online. 2013;18:21877.
Advances in Medical Education and Practice

\section{Publish your work in this journal}

Advances in Medical Education and Practice is an international, peerreviewed, open access journal that aims to present and publish research on Medical Education covering medical, dental, nursing and allied health care professional education. The journal covers undergraduate education, postgraduate training and continuing medical education

\section{Dovepress}

including emerging trends and innovative models linking education, research, and health care services. The manuscript management system is completely online and includes a very quick and fair peer-review system. Visit http://www.dovepress.com/testimonials.php to read real quotes from published authors. 